\title{
Utilizing Alternative Testing Technology for Human Immunodeficiency Virus (HIV) in the COVID era
}

\author{
Isabella Warmbrunn ${ }^{1}$ (D) $\cdot$ Monique Green-Jones ${ }^{1} \cdot$ Angulique Y. Outlaw $^{1}$ \\ Accepted: 23 March 2021 / Published online: 5 May 2021 \\ (C) The Author(s), under exclusive licence to Springer Science+Business Media, LLC, part of Springer Nature 2021
}

\begin{abstract}
Purpose of Review With the onset of the Coronavirus disease 2019 (COVID-19) pandemic, in-person human immunodeficiency virus (HIV) testing is no longer easily accessible. Therefore, alternative testing technologies must be considered and implemented on a large scale to continue prevention efforts. This review seeks to describe the benefits of utilizing at-home HIV testing technologies, traditionally deemed an alternative form of testing.

Recent Findings Utilizing at-home testing technology during the COVID-19 pandemic overcomes novel and previously identified barriers to HIV testing.

Summary Ensuring access to HIV testing is imperative to long-term prevention goals. With the Ending the HIV Epidemic (EHE) initiative in the USA targeting achievement by 2030, obstacles to HIV testing must be addressed to ensure its success. Implementing alternative testing technology more broadly allows for continued prevention efforts for HIV in light of COVID era restrictions.
\end{abstract}

Keywords HIV $\cdot$ Self-testing $\cdot$ COVID-19 $\cdot$ Testing technology $\cdot$ Prevention $\cdot$ Sexual health

\section{Introduction}

In 2018, approximately 36,400 new human immunodeficiency virus (HIV) infections were diagnosed in the United States (US) alone [1]. Through decades of HIV prevention research, the scientific community has learned that screening is integral to managing the spread of the virus. The Centers for Disease Control and Prevention (CDC) recommends that everyone between the ages of 13 and 64 be screened for HIV at least once during a routine care visit [2]. The recommended frequency of HIV screening increases with engagement in higher risk behaviors such as follows: sex with multiple partners, condomless sex with someone who is living with HIV or whose HIV status is unknown, and injecting drugs with shared needles $[2,3]$. These behaviors appear to increase both exposure to the virus and the risk of contracting HIV infection.

This article is part of the Topical Collection on Technology in Medicine

Isabella Warmbrunn

fv2676@wayne.edu

1 Department of Family Medicine and Public Health Sciences, Wayne State University School of Medicine, Detroit, MI, USA
It is estimated that $69 \%$ of all new HIV infections occur among men having sex with men (MSM) [4]. The CDC recommends that sexually active MSM should be tested for HIV at least once per year, and encourages men with additional high-risk factors to test as often as every 3-6 months [5]. Even under pre-COVID conditions, it has been shown that many MSM do not adhere to this CDC-recommended testing schedule $[6,7]$. Recent limitations in access to traditional forms of HIV place MSM at an even greater risk [8•]. The dearth of available in-person HIV testing may exacerbate already decreased MSM compliance with preventative clinic visits. During many of these preventative visits, access to additional services and supplies are provided. These additional services can include pre-exposure prophylaxis (PrEP) referrals, sexually transmitted infection (STI) testing, and provision of free condoms. Thus, the inability to receive in-person HIV testing not only limits knowledge of HIV status, but can also reduce access to services and supplies integral to the prevention of HIV transmission within high-risk populations.

There are two CDC-approved forms of HIV testing: traditional in-person testing at a designated clinic or at a primary care appointment, and novel at-home testing. Traditional barriers to in-person testing include access to testing services, the cost of test, and wait time [6,9-12]. These barriers were 
considered in the Federal Drug Administration (FDA) decision to approve the OraQuick® In-Home HIV Test in 2012. The OraQuick $®$ test differs from traditional HIV testing technologies, as it is completed with an oral swab test and can be completed without assistance at home. Like rapid HIV testing technologies, results are available in about $20 \mathrm{~min}$. By comparison, traditional in-person testing utilizes a blood sample, and depending on the test type, the wait time for the result varies from $20 \mathrm{~min}$ (for a rapid finger prick test) to 5 days for (laboratory-based tests) [13]. Access to two forms of testing technology, in-person and at-home, ensures that individuals have access to regular screening and timely awareness of their HIV status.

\section{Traditional Barriers to HIV Testing}

Many of the current barriers to HIV testing were present prior to COVID-19. Community-based HIV testing locations are typically centralized in high-density urban areas which are not always accessible to a larger demographic. The cost for an in-person HIV test can vary with most community-based organizations $(\mathrm{CBO})$ providing free rapid tests, and non-CBO (such as a doctor's office or clinics) using traditional lab-based testing that may not be covered fully depending on insurance status. For preventative services, most insurance companies cover the cost of an annual HIV test; after this, insurance coverage can vary if HIV testing has been completed previously in the same year [14]. Additionally, result times of labbased testing can vary from 48 to $72 \mathrm{~h}$ for results to be completed. The precise duration of time is contingent on the location of the office in proximity to the laboratory that conducts the analysis.

These previous barriers to HIV testing are mostly addressed with the approval of OraQuick® in 2012. Rapid testing with OraQuick ${ }^{\circledR}$ enables individuals to see highly accurate test results (over $90 \%$ test sensitivity) in $20 \mathrm{~min}$, given no user error [15-18]. The OraQuick ${ }^{\circledR}$ tests are offered at major retail locations for fixed prices ranging from $\$ 40$ to 50 , and these costs can be mitigated if provided from a CBO. The ease of access, fixed price, speed of results, and accuracy of the testing technology make utilizing at-home testing an ideal solution to barriers to HIV testing.

\section{Obstacles to HIV Testing in the COVID-19 Era}

The availability of in-person HIV testing fundamentally changed with the onset of the coronavirus disease 2019 (COVID-19). Following the World Health Organization (WHO) announcement that COVID-19 was a pandemic, many non-essential healthcare facilities were temporarily closed as a safety precaution. Prioritization of containing and preventing the spread of COVID-19 disease resulted in lockdowns of many centers deemed not life-sustaining or medi-

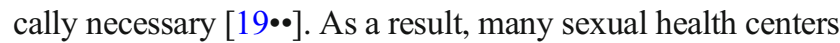
providing HIV and other sexual health screenings are temporarily closed [19••].

As a result of these temporary closings, the largest obstacle for maintaining HIV prevention services during COVID era restrictions has been the inability to conduct in-person HIV testing. This traditional form of HIV testing has been utilized in many community-based clinics, usually at no charge, to facilitate community members' knowledge of their HIV status. These restrictions do not just limit access to in-person community-based testing; access to other preventative services, such as the prescription of pre-exposure prophylaxis (PrEP), has also been severely limited. The prescription of PrEP medication is often indicated for those at risk for HIV transmission from sexual intercourse or injection drug use. Daily pill of Truvada ${ }^{\circledR}$ (emtricitabine/tenofovir disoproxil fumarate) or Descovy ${ }^{\circledR}$ (emtricitabine \& tenofovir alafenamide) has been shown to be over $90 \%$ effective in preventing HIV transmission via sexual intercourse [20]. COVID-19 restrictions also affect the availability of clinicians who are responsible for discussing PrEP treatment plans and concerns regarding HIV treatment regimens with patients [19••]. Due to the demands of the COVID-19 pandemic, many clinicians were called to work in critical care units or directly with COVID-19 patients. This decreases the number of physicians who can work with HIV clients, directly affecting prescribing PrEP as a tool to prevent the transmission of HIV [21].

Recent studies have shown that the temporary closure of clinics and community drop-in sexual health centers due to COVID-19 pandemic has resulted in a significant portion of the population being unable to receive in-person HIV testing $[22 \cdot, 23]$. This has made traditional in-person testing an impossibility for many at-risk individuals. This is especially problematic considering that an estimated $40 \%$ of all new HIV transmissions involve individuals who were recently infected, but were unaware of their HIV-positive status [2]. A call to action from Shoptaw et al. states the importance of scaling up access to at-home HIV testing to better inform atrisk individuals of their HIV status in the COVID-19 era [19••, 23].

\section{Sexual Activity During COVID-19}

In an online survey of 1051 MSM located in the US, Sanchez et al. reported that approximately half $(48 \%)$ of their survey participants reported no change in their number of sex partners during the COVID-19 pandemic [24••]. Also, many reported unaffected usage of dating or hook-up apps to connect or meet with other men $[24 \cdot \bullet]$. These findings are confirmed in an international survey distributed online via social media from 
March to April 2020. Results indicated that sexual intercourse had not been affected within English or Spanish-speaking populations [25*0]. Previous studies have correlated sex with increased satisfaction with one's mental health and overall quality of life $[24 \cdot \bullet, 25 \bullet \cdot]$. So, it is understandable that sexual intercourse can mitigate the higher levels of stress, anxiety, and isolation associated with the severity and duration of the COVID-19 pandemic [26]. However, the limitation of inperson HIV testing creates additional risks and stressors for unknowingly transmitting HIV to a sexual partner. This is especially concerning as MSM sexual activity has remained relatively constant during COVID-19 pandemic, suggesting that the level of risk HIV transmission likely remains at least as high as it was prior to the pandemic [25••].

\section{OraQuick $^{\circledast}$ Technology}

The HIV self-test (ST) has been utilized to fill the gaps in access to traditional testing services. As of 2019, over 77 countries have adopted some form of ST policy [27]. At present, OraQuick ${ }^{\circledR}$ is the only FDA-approved rapid oral ST. OraSure; the manufacture of OraQuick $®$ has decades of experience in creating HIV testing technologies. In 1994, they obtained approval for the first oral specimen collection device for HIV-1 to be used in tandem with laboratory testing. Shortly after, in 2002, the first rapid HIV-1 test was developed and approved in the US by the FDA. The OraQuick® rest, approved by the FDA in 2012 , is a single-use, qualitative immunoassay that can be purchased in retail locations nationwide [28]. This test detects the presence of HIV antibodies and not the virus itself; which is why the results are primary in nature and need confirmatory laboratory-based testing. The testing kit contains an oral swab, tube with buffered solution, step-by-step instruction pamphlet, and information booklet on HIV. The testing kit also includes a package insert containing information about the test technology, and a toll-free number to call with questions during and after the testing process. The website for OraQuick ${ }^{\circledR}$ also contains a walkthrough video demonstrating how to properly complete the test, as well as alternative ways to link individuals to care after results are rendered (Fig. 1) [15, 28].

The test is completed by running the oral swab across the upper and lower gums once and then inserting it into the fluid- filled test tube and marking the completion time [27]. After 20 min, the results will appear on the $\mathrm{C}$ and/or $\mathrm{T}$ line. A line next to the $\mathrm{C}$ indicates that the test is working; no line next to the $\mathrm{C}$ indicates that the test is defective and the results are invalid. A line on $\mathrm{C}$ but not $\mathrm{T}$ indicates a negative result, and a line on $\mathrm{C}$ and $\mathrm{T}$ indicates a positive result [29]. Test results can be inaccurate if viewed $40 \mathrm{~min}$ after starting and will need to be redone for an accurate interpretation [18]. The manufacturer recommends that confirmation of negative results with labbased testing are needed prior to starting PrEP treatment. Negative results are likely accurate if it has been 3 months or longer since engaging in any high-risk events [29]. A positive result must be confirmed by a clinic or healthcare professional. The OraQuick ${ }^{\circledR}$ website and testing booklet provide access to local resources and a toll-free number to obtain assistance with next steps [30, 31].

OraQuick ${ }^{\circledR}$ has been clinically studied for correctly indicating HIV status and has maintained a $92 \%$ and $99.98 \%$ accuracy for positive and negative results, respectively [32]. In other words, for every 5000 uninfected individuals, there is one false positive. It is recommended that individuals with a negative result follow-up with testing as the "window period" required for antibodies to form can be up to 3 months postexposure [33].

\section{Acceptance of HIV ST}

Based upon the existing evidence, HIV ST appears to be a viable alternative to traditional in-person testing. There has already been demonstrated preference for HIV self-testing among MSM [16, 34••]. Over the past 5 years, many national and international agencies have adjusted guidelines to be more inclusive of alternative HIV screening strategies [22•, 23, 35]. In 2016, the WHO issued a strong recommendation for ST as an additional approach to HIV testing services [34••]. As of May 2020, the National Center for HIV/AIDS, Viral Hepatitis, STD, and TB Prevention (NCHHSTP) recommends the use of HIV ST for CBO and health departments as a as a means of providing prevention services to residents [22•]. The NCHHSTP also issued a statement in support of expanding HIV screening strategies, such as HIV self-testing, to assist in adapting to current COVID-19 restrictions [23]. In the event that laboratory-based testing is unavailable, new
Fig. 1 Result strip of the OraQuick® In-Home HIV Test (reprinted with permission from OraSure Technologies, Inc.)

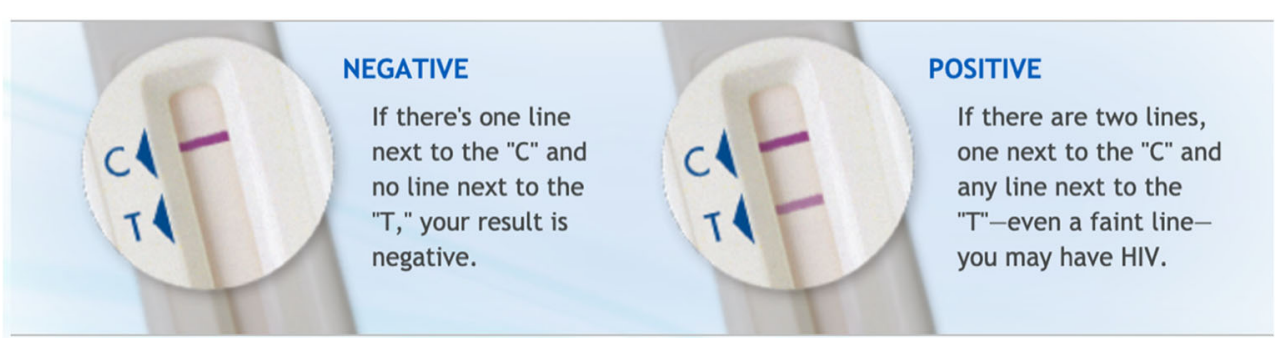


recommendations from the $\mathrm{CDC}$ enable the provision of a PrEP prescription with a non-reactive HIV ST [35]. This will ensure that at-risk individuals can access this needed medication for protection from the HIV infection during the COVID19 pandemic.

In addition to these changes to national guidelines, state and local organizations have also promoted increased use of HIV ST technologies. One such measure is the partnership of the Michigan Department of Human and Health Services (MDHHS) with a Detroit-based agency, Wayne State University Prevention Team (W'SUP), to pilot on-demand mail delivery of HIV ST kits to individuals ages 18-34 [36]. By targeting sexually active, Wayne county residents aged 18-34, this initiative targets a major at-risk demographic for HIV transmission [37]. This program has been prompted by the high rate of HIV cases in Wayne County, with Wayne and Oakland county making up the highest rates of persons living with HIV in the state [38].

Programming like this can be integrated into pre-existing funded programming, and clinics that are currently unable to provide in-person services may be able to offer free or lowcost HIV ST kit as a temporizing measure to facilitate testing within their communities.

Prior to COVID-19, it was reported that access to HIV selftesting could improve HIV testing within at-risk demographics, many of whom reside in inner cities. In an urban setting, up to $60 \%$ of patients decline routine HIV testing offered during an emergency department (ED) visit [39]. As reported by Patel et al. in 2018, one solution is offering HIV ST kits to those who decline HIV testing in the ED [40]. Participants reported that confidentiality, privacy, and comfort of their own home were driving factors for self-testing engagement [40]. These reasons are even more applicable with the risk of COVID-19 exposure in public settings. These results suggest that HIV ST can lead to an increase in HIV testing rates from a commonly missed at-risk demographic despite COVID-19 era restrictions.

\section{Linkage to PrEP}

With PrEP referrals reliant on first obtaining a negative HIV test, the inability to access commonly used in-person HIV testing services makes initiating PrEP more difficult. PrEP use is recommended for HIV-negative individuals who engage in higher-risk sexual activity, as it significantly decreases their risk of contracting HIV [41]. Most PrEP referrals are provided during an in-person HIV testing appointment by the test counselor or prescribed by a physician during a medical visit. However, PrEP is most effective when taken in concert with scheduled medical visits every 3 months for HIV and other biomarker testing [20, 42]. The COVID-19 restrictions on in-person clinic visits for routine HIV testing and other difficulties obtaining a non-emergent visit with a physician severely impair this prescription and referral of PrEP. This results in a large demographic being unable to access PrEP medications. The use of HIV self-testing could alleviate this disparity, as HIV self-testing kits could be sent out to individuals who are on PrEP every 3 months. This would enable PrEP users to frequently confirm their current HIV-negative status, which is critical to continued use of PrEP, without the risk of exposure to COVID-19 that occurs when traveling to a clinic or hospital for laboratory-based HIV testing. Distribution of STs also enables those interested in starting PrEP the linkages to local healthcare providers who can remotely work with them on properly maintaining their PrEP regimen.

\section{STI Testing and Free Condoms}

In an online survey from Sanchez et al., younger MSM participants reported more difficulties accessing HIV and sexually transmitted infection (STI) testing, as well as treatment services during COVID-19 pandemic [24••]. These limitations of access can be overcome with HIV ST. However, full access to other forms of HIV prevention (e.g., STI testing, free condoms) is limited. It is possible that by utilizing HIV ST, individuals can be linked to local operating sexual health clinics where they can make an immediate appointment and obtain an in-person STI test. This method is likely faster than the alternative of requesting an STI ST kit, which can have more drawbacks. Like HIV ST, self-testing for STI is also available; however, there are more factors to consider such as follows: speed of results, and cost of the STI ST kit. Results from an at-home STI ST kit are not rapidly reported and often involve collecting blood, urine, oral, and anal specimens that must be mailed back to the laboratory for analysis. Additionally, the cost of these kits can be considerable and not often able to be absorbed by the budgets of CBO. Most STI ST kits are offered by mail-order laboratories and can be customized, which will influence the price. These limitations for STI self-testing are not seen in HIV self-testing, and with use of an HIV ST, it is possible to be linked more rapidly for STI testing if needed.

In addition to testing services, many sexual health clinics and CBO provide free sexual health supplies such as condoms, dental dams, and lubricant. With current COVID-19 travel and lockdown restrictions, access to these necessary supplies remains limited. In a recent study by Stephenson et al., approximately $30 \%$ of participants reported that COVID-19 restrictions prevented from HIV or STI testing [43]. Accessibility to sexual health supplies is adapting, with some local health departments providing free condoms to residents. In Detroit, residents can request condoms to be directly 
mailed to their home address on the city's health department website [44].

\section{HIV ST Programs}

National programs for providing free at-home HIV ST kits are already underway. Greater than AIDS, a public HIV information response from the non-profit Kaiser Family Foundation has partnered with Walgreens and OraSure Technologies. This partnership has enabled 10,000 OraQuick ${ }^{\circledR}$ tests to be provided at no-cost to various local health agencies and CBO across the US [45]. In Michigan, W'SUP and MDHHS have partnered to mail HIV ST kits on-demand to eligible individuals. In Ohio, the University of Miami Developmental HIV/ AIDS Mental Health Research Center has launched a project to provide free HIV testing and same day start packet of HIV medications in mobile syringe service programs [46]. Yalefunded researchers are collaborating with researchers in Puerto Rico to learn how to increase at-home HIV testing [19••]. These are all steps towards the future evolution of HIV prevention despite COVID era restrictions.

It is important to note that HIV self-testing is not a perfect solution. Limitations and drawbacks exist, including limited follow-up and lack of engagement from a healthcare provider during the testing process. Receiving a positive result can be overwhelming, and those found to have a reactive ST must be linked to a healthcare provider for confirmatory laboratorybased testing as well as other resources to provide medical, psychological, and emotional support. The need for more active follow-up must be considered an integral part of this scheme [34••]. Video conference with an HIV testing counselor or healthcare provider when waiting or reviewing HIV ST results may be helpful.

Providing uninterrupted testing of at-risk individuals is needed to ensure timely access to preventative and therapeutic interventions to prevent HIV transmission. The duration of travel and care restrictions due to the COVID-19 pandemic remains unclear, but the path to prevention of future transmission of HIV is not. Despite its limitations, self-testing for HIV is a viable part of the solution going forward. With the intention to "End the HIV Epidemic in the United States by 2030," proactive efforts, like HIV ST, are needed [47]. Funding from national and local institutions to enhance the availability of HIV self-testing and follow-up care may help to ensure meeting this goal, despite current obstacles to traditional in-person HIV testing methods.

\section{Conclusion}

Considering current travel and lockdown restrictions due to COVID-19, the reliance on traditional methods to prevent
HIV transmission may not be adequate. This pandemic has shown that the traditional model for HIV testing, which is contingent on accessibility to in-person HIV testing, must undergo an evolution. With limited access to in-person testing sites, at-home HIV ST may be the ideal solution. Increased use of HIV ST may address previous barriers to HIV testing including potential test cost, access to testing location, and speed of results. Like serum HIV tests, HIV self-testing has demonstrated high reliability and accuracy. However, providers must understand the limitations of the current technology, as well as the need for improved post-testing care and patient counseling. Progress has already been made in the effort to provide affordable HIV ST to higher risk individuals, and this approach has already been shown to be popular with target populations. Additional effort is needed to ensure that the COVID-19 pandemic does not further undermine prevention efforts to end the HIV epidemic.

\section{Declaration}

Human and Animal Rights and Informed Consent This article does not contain any studies with human or animal subjects performed by any of the authors.

Conflict of Interest The authors declare no competing interests.

\section{References}

Papers of particular interest, published recently, have been highlighted as:

- Of importance

•- Of major importance

1. Centers for Disease Control and Prevention. Estimated HIV incidence and prevalence in the United States, 2014-2018. HIV Surveillance Supplemental Report 2020;25(No. 1). http://www. cdc.gov/hiv/library/reports/hiv-surveillance.html. Published May 2020.

2. HIV Testing | HIV/AIDS | CDC [Internet]. CDC.gov. 2021. Available from: https://www.cdc.gov/hiv/testing/index.html

3. HIV Risk Behaviors | HIV Risk and Prevention Estimates | HIV Risk and Prevention | HIV/AIDS |CDC [Internet]. CDC.gov. 2021. Available from: https://www.cdc.gov/hiv/risk/estimates/ riskbehaviors.html

4. Centers for Disease Control and Prevention. HIV Surveillance Report. 2018 (Updated); vol. 31. http://www.cdc.gov/hiv/library/ reports/hiv-surveillance.html. Published May 2020.

5. DiNenno EA, Prejean J, Irwin K, et al. Recommendations for HIV screening of gay, bisexual, and other men who have sex with men — United States, 2017. MMWR Morb Mortal Wkly Rep. 2017;66: 830-2. https://doi.org/10.15585/mmwr.mm6631a3.

6. Campbell C, Lippman S, Moss N, Lightfoot M. Strategies to increase HIV testing among MSM: a synthesis of the literature. AIDS Behav. 2018;22:2387-412.

7. Hall E, Ricca A, Khosropour C, Sullivan P. Capturing HIV incidence among MSM through at-home and self-reported facility- 
based testing. JAIDS Journal of Acquired Immune Deficiency Syndromes. 2017;75:e142-4.

8. Shilo G, Mor Z. COVID-19 and the changes in the sexual behavior of men who have sex with men: results of an online survey. J Sex Med. 2020;17(10):1827-34 An international study confirming national studies on sexual behavior trends during the COVID-19 pandemic.

9. Wise J, Ott C, Azuero A, Lanzi R, Davies S, Gardner A, et al. Barriers to HIV testing: patient and provider perspectives in the deep south. AIDS Behav. 2019;23:1062-72.

10. Schwarcz S, Richards T, Frank H, Wenzel C, Chin Hsu L, Chin C, et al. Identifying barriers to HIV testing: personal and contextual factors associated with late HIV testing. AIDS Care. 2011;23: 892-900.

11. Syed S, Gerber B, Sharp L. Traveling towards disease: transportation barriers to health care access. J Community Health. 2013;38: 976-93.

12. National Center for HIV/AIDS, Viral Hepatitis, STD, and TB Prevention Fact Sheet: HIV testing in the United States, 2016 [Internet]. Centers for Disease Control and Prevention; 2016. Available from https:/www.cdc.gov/nchhstp/newsroom/docs/ factsheets/hiv-testing-us-508.pdf

13. Cdc.gov. Types of HIV Tests | Testing | HIV Basics | HIV/AIDS | CDC. 2020. [online] Available at: https://www.cdc.gov/hiv/basics/ hiv-testing/test-types.html

14. Michigan Quality Improvement Consortium. Adult Prevention Services (18-49) [Internet]. [Michigan]: MQIC; 2019. (Clinical guideline). Available from http://mqic.org/guidelines.htm

15. OraQuick ${ }^{\circledR}$ Video for In Home HIV Test Kit [Internet]. OraQuick®.com. 2021 [cited 8 March 2021]. Available from: http:/www.OraQuick®.com/Taking-the-Test/How-To-Video

16. Oral HIV Testing at Home OraQuick ${ }^{\circledR}$ [Internet]. OraQuick®.com. 2021 [cited 8 March 2021]. Available from: http://www.OraQuick®.com

17. Centers for Disease Control and Prevention. Rapid HIV tests suitable for use in non-clinical settings (CLIA-waived), 2018. Publicuse data file and documentation https://www.cdc.gov/hiv/pdf/ testing/rapid-hiv-tests-non-clinical.pdf

18. Center for Biologics Evaluation and Research. (n.d.). Information regarding the OraQuick In-Home HIV Test [Internet]. U.S. Food and Drug Administration. FDA; Available from: https://www.fda. gov/vaccines-blood-biologics/approved-blood-products/ information-regarding-oraquick-home-hiv-test

19.• Shoptaw S, Goodman-Meza D, Landovitz R. Collective call to action for HIV/AIDS community-based collaborative science in the era of COVID-19. AIDS Behav. 2020;24:2013-6 Addressing novel strategies to continue HIV prevention efforts in the COVID-era.

20. PrEP Effectiveness | PrEP | HIV Basics | HIV/AIDS | CDC [Internet]. CDC.gov. 2021 [cited 8 March 2021]. Available from: https://www.cdc.gov/hiv/basics/prep/prep-effectiveness.html

21. Eakle R, Venter F, Rees H. Pre-exposure prophylaxis (PrEP) in an era of stalled HIV prevention: can it change the game? Retrovirology. 2018;15.

22. HIV Self Testing Guidance April 28, $2020|2020|$ Dear Colleague Letters | NCHHSTP | CDC [Internet]. CDC.gov. 2021 [cited 8 March 2021]. Available from: https://www.cdc.gov/nchhstp/dear colleague/2020/dcl-042820-HIV-self-testing-guidance.html. $\overline{\mathbf{A}}$ letter of support from CDC Division of HIV/AIDS Prevention for HIV self-testing in during the COVID-19 pandemic.

23. National HIV Testing Day June 25, 2020 | 2020 | Dear Colleague Letters | NCHHSTP | CDC [Internet]. CDC.gov. 2021 [cited 8 March 2021]. Available from: https://www.cdc.gov/nchhstp/dear colleague/2020/dcl-062520-NHTD.html

24.• Sanchez TH, Zlotorzynska M, Rai M, Baral SD. Characterizing the impact of COVID-19 on men who have sex with men across the
United States in April, 2020. AIDS Behav. 2020;24:2024-32. https://doi.org/10.1007/s10461-020-02894-2 Demonstrates the impact of COVID-19 on a large at-risk demographic for HIV in the United States.

25.• Ibarra FP, Mehrad M, Di Mauro M, MFP G, Cruz EG, Nilforoushzadeh MA, et al. Impact of the COVID-19 pandemic on the sexual behavior of the population. The vision of the east and the west. Int Braz J Urol. 2020;46(suppl.1):104-12. https://doi.org/ 10.1590/S1677-5538.IBJU.2020 S116. Discussion on how COVID-19 pandemic has affected sexual behavior internationally. The results highlight the importance to implement alternative forms of HIV testing.

26. CDC. COVID-19 and Your Health [Internet]. Cdc.gov. 2021. Available from: https://www.cdc.gov/coronavirus/2019-ncov/ daily-life-coping/managing-stress-anxiety.html

27. WHO | HIV self-testing. 2019. Available from: https://www.who. int/hiv/topics/self-testing/en

28. Where to Buy OraQuick ${ }^{\circledR}$ In Home HIV Test Kits [Internet]. OraQuick®.com. 2021 [cited 8 March 2021]. Available from: http:/www.OraQuick®.com/where-to-buy

29. Orasure Technologies. OraQuick ${ }^{\circledR}$ In-home HIV Test- How-to Video. 2012. https://www.youtube.com/watch?v= 010yO9iQYOc\&ab channel=OraSureTechnologies

30. OraQuick®.com. 2021. OraQuick ${ }^{\circledR}$ results for in home HIV testing. [Internet] Available at: http://www.OraQuick®.com/ Taking-the-Test/Understanding-Your-Results.

31. HIV FAQ | OraQuick ${ }^{\circledR}$ Home HIV Test [Internet]. OraQuick®.com. 2021 [cited 8 March 2021]. Available from: http://www.OraQuick®.com/FAQs

32. Information regarding the OraQuick ${ }^{\circledR}$ In-Home HIV Test [Internet]. U.S. Food and Drug Administration. 2021 [cited 8 March 2021]. Available from: https://www.fda.gov/vaccinesblood-biologics/approved-blood-products/information-regardingOraQuick®-home-hiv-test.

33. Types of HIV Tests | Testing | HIV Basics | HIV/AIDS | CDC [Internet]. Cdc.gov. 2021 [cited 8 March 2021]. Available from: https://www.cdc.gov/hiv/basics/hiv-testing/test-types.html

34.• Wray T, Chan P, Simpanen E, Operario D. eTEST: developing a smart home HIV testing kit that enables active, real-time follow-up and referral after testing. JMIR mHealth and uHealth. 2017;5:e62 Discussion of the importance of follow-up and referral after self-testing.

35. Maksut J, Eaton L, Siembida E, Driffin D, Baldwin R. A test of concept study of at-home, self-administered HIV testing with webbased peer counseling via video chat for men who have sex with men. JMIR Public Health Surveill. 2016;2:e170.

36. Step-by-step [Internet]. MiHIV status. 2021. Available from: https:// www.mihivstatus.org/step-by-step

37. HIV Rapid Testing and Education: Midtown Detroit: WSUP [Internet]. wsupdet. [2021]. Available from: https://www. wsup313.com/

38. Michigan Department of Health and Human Services. Detroit Metro Area HIV Surveillance Report New Diagnoses and Prevalence Tables, 2018. https://www.michigan.gov/documents/ mdhhs/DMA HIV Surveillance Report - July 2018633934 7.pdf

39. PrEP During COVID-19 | $2020 \mid$ Dear Colleague Letters NCHHSTP | CDC [Internet]. Cdc.gov. 2021 [cited 8 March 2021]. Available from: https://www.cdc.gov/nchhstp/dear colleague/2020/dcl-051520-PrEP-during-COVID-19.html

40. Haukoos J, Lyons M, Rothman R, et al. The HIV tested trial: a multi-center pragmatic randomized comparison of HIV screening strategy effectiveness in the emergency department. Acad Emerg Med. 2017;24(S1):S42-3.

41. Patel A, Abrams S, Gaydos C, Jett-Goheen M, Latkin C, Rothman $\mathrm{R}$, et al. Increasing HIV testing engagement through provision of 
home HIV self-testing kits for patients who decline testing in the emergency department: a pilot randomisation study. Sex Transm Infect. 2018;95:358-60.

42. Pre-Exposure Prophylaxis [Internet]. HIV.gov. 2020 [cited 8 March 2021]. Available from: https://www.hiv.gov/hiv-basics/ hiv-prevention/using-hiv-medication-to-reduce-risk/pre-exposureprophylaxis

43. Stephenson R, Chavanduka T, Rosso MT, Sullivan SP, Pitter RA, Hunter AS, et al. Sex in the time of COVID-19: results of an online survey of gay, bisexual and other men who have sex with men's experience of sex and HIV prevention during the US COVID-19 epidemic. AIDS and behavior:1-9. Advance online publication. https://doi.org/10.1007/s10461-020-03024-8.

44. Order Free Condoms by Mail | City of Detroit [Internet]. City of Detroit. 2021. Available from: https://detroitmi.gov/departments/ detroit-health-department/programs-and-services/stdhivprevention/order-free-condoms-mail

45. Free HIV Self-Test [Internet]. Greater Than AIDS. 2021. Available from: https://www.greaterthan.org/free-hiv-self-test/
46. University of Miami Developmental HIV/AIDS Mental Health Research Center (D-ARC) [Internet]. University of Miami's Research Profiles. 2021. Available from: https://miami.pure. elsevier.com/en/projects/university-of-miami-developmentalhivaids-mental-health-research-

47. Ending the HIV Epidemic in the United States [Internet]. Official web site of the U.S. Health Resources \& Services Administration. 2021 [cited 8 March 2021]. Available from: https://www.hrsa.gov/ ending-hiv-epidemic

48. HIV Counseling | OraQuick ${ }^{\circledR}$ Home HIV Test [Internet]. OraQuick®.com. 2021 [cited 8 March 2021]. Available from: http://www.OraQuick®.com/Find-Counseling-and-Care

49. Riddell J, Amico K, Mayer K. HIV preexposure prophylaxis. JAMA. 2018;319:1261.

Publisher's Note Springer Nature remains neutral with regard to jurisdictional claims in published maps and institutional affiliations. 